\title{
Science Investigation System for Telescopes in Education Research
}

\author{
Susan Hoban \\ $U M B C^{*}$ \\ John Keating \\ NUIM** \\ Joel Sachs \\ $U M B C$ \\ hoban@umbc.edu \\ john.keating@may.ie \\ jsachs@csee.umbc.edu \\ Daniel Laughlin
UMBC
dlaugh@umbc.edu \\ Yelena Yesha \\ $U M B C$ \\ yeyesha@csee.umbc.edu \\ *University of Maryland Baltimore County \\ **National University of Ireland Maynooth
}

\begin{abstract}
The Virtual Telescopes in Education (VTIE) project, is providing tools for teaching astronomy in $K-12$ classrooms using remotely controlled telescopes. VTIE's main goal to promote scientific literacy, including a conceptual understanding of the physical world and the scientific process itself. This paper describes an essential component of VTIE: the Scientific Investigation System for Telescopes in Education Research (SISTER). Students are aided in the preparation of their observing proposal by a novel Glossary Tool for dynamically inducing hyperlinks tailored to a student's reading level, a. Proposal Generation Interface to guide them through the process of formulating a scientific question, and a Paper Writing Tool to report the results of the analysis of the data. In this paper we present the architecture and methodology for SISTER..
\end{abstract}

\section{Introduction}

The Virtual Telescopes in Education (VTIE, http://vtie.gsfc.nasa.gov) project is developing the services required to operate a network of robotic telescopes for use in education, including a scheduling service, an active data archive, tools for data manipulation, an online proposal preparation and review environment, an online VTIE Journal for publication of results, and access to related educational materials provided by the educational community. VTIE is a partner with the Telescopes in Education project (http://tie.jpl.nasa.gov) though the software under development is generic and not tied to any particular hardware platform or operating system.

The use of remotely controlled and automated telescopes for K-12 education is no longer novel. Since 1993, Telescopes in Education has been providing telescope access to classrooms and is currently working with approximately 20 observatories world-wide [1]. The Bradford Robotic Telescope Observatory (http://www.telescope.org) has been operating for nearly a decade [2] and is used by teachers and students in Great Britain. In 2001, the Charles Sturt University Remote Telescope (http://www.csu.edu.au/telescope/) launched $A$ Journey Through Space and Time, a project for primary and secondary students in New South Wales, Australia.

The philosophies differ among the designers of the systems. In the case of automated telescopes such as the Bradford telescope, the goal is to maximize the field of potential users of the data, so the telescope takes data whenever the prevailing weather conditions permit. Students submit their requests, and retrieve the data the morning after their data have been obtained; this mode of operation is referred to as Service Observing in the astronomy community [3]. Service Observing obviates the need for students to stay up late at night and does not require students, teachers and parents to arrange their schedules around the fickle finger of the weather. Remotely controlled telescopes, on the other hand, such as those used by Telescopes in Education and A Journey Through Space and Time, allow the students to interact with the telescope directly from a remote location (Bradford also offers this mode of operation, but it is not the standard process). This active participation in the observations is considered a critical part of the process [4], both from an inspirational perspective and from the perspective of problem-based learning approach (see e.g. [5]). Telescopes in Education has freed its users from the day-night constraints driven by their geographic location by engaging partners at various longitudes around the globe. The VTIE project is, in some sense, merging the philosophies described above. VTIE is providing a constraint-based scheduler [6] built on an agent-based resource management system [7] to maximize the use of 
available telescope time in the network. At the same time, VTIE is strongly supporting the move from "star parties to science" by providing software to guide students in the development of a science project in a multi-media, problem-based learning environment. Problem-based learning, particularly in multi-media environments, has been shown to be an effective way to engage students (e.g. [8]; [9]), potentially leading to a sense of ownership and investment that helps student internalize the educational goals of an assignment. By making them partners in the process of their own education, they are more likely to delve into deeper levels of understanding. Providing students with realistic situations and guiding them through the scaffolding of the structured questions allows them to approach the same sort of problems with which astronomers deal. Explicit training with problem-based learning procedures often takes several classes [10]. The guidance in forming questions that can be answered by the range of available observations provided by the proposal generation tool allows students to start doing the work of problem solving without having to learn problem-based learning strategies before hand. In turn, the questions generated by the students using the proposal generation tool provide them with models of how to structure questions in a scientific investigation. The nature of the information available limits the possible approaches for finding answers. While that limit means there is not total freedom in the investigation, it both realistically narrows the possibilities and helps insure that students will be able to identify an approach to the problem that is likely to be successful.

The problem-based learning approach of VTIE takes students up the ladder of Bloom's taxonomy [11] from concrete, lower-order thinking about concrete information to higher-order analysis and synthesis. Students go well beyond the level of reading in a textbook or on the web about how astronomy is done. They are introduced to the process doing authentic, realworld tasks. The students learn and use cognitive skills that are of general use [12] and more particularly for VTIE, teach them about the process of science.

Upon completion, the VTIE products will complement the work of existing efforts that use telescopes to bring real astronomy into the K-12 classroom. This paper describes an essential component of VTIE: the Scientific Investigation System for Telescopes in Education Research (SISTER). SISTER is a suite of learning tools that assists students in the preparation of an observing proposal and guides the students through the reporting of their results, culminating in the online VTIE Journal.

\section{The SISTER Process}

The SISTER process, enclosed inside the dashed box shown in Figure 1, uses a suite of tools seamlessly integrated within the VTIE portal which provide a framework to support student participation in the science process. Astronomy is used as the example for implementation of the framework. SISTER guides the students through the problem-based learning framework as they embark upon their discovery of properties of celestial sources.

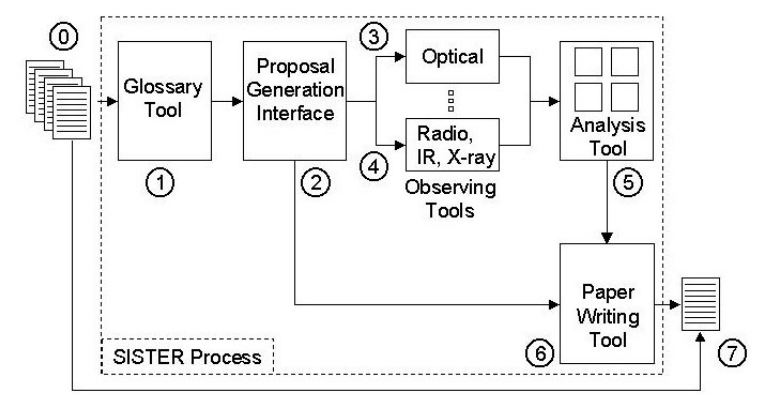

Figure 1: SISTER Process

The desired outcome using SISTER that VTIE strives to achieve is the enhancement and refinement of students' ability to design and carry out experimental procedures to address questions about astronomy through measurement and analysis of astronomical sources. One can consider the inputs to SISTER to be the extant set of resources pertaining to astronomy, on the Web, in textbooks, magazines, etc., as well as the lesson plans and assistance provided by the teacher (Item 0 in Figure 1). The output of the process is the student's report: a written description of the procedures, analysis methods and results (Item 7 in Figure 1), which is stored in the VTIE Journal. The approach provides a structured process that encapsulates many online tools, and may be 'paused' and 'resumed' from class-to-class with ease.

The first step in the process requires the teacher to provide or identify suitable background reading and resources for students to develop concepts about astronomy so that they may begin to formulate questions. The next step is for the students to refine the question so that it can be addressed by an observation of an astronomical object with one of the telescopes available to them. The students then obtain the images and analyze them. They draw conclusions from the analysis, and finally they write a report on their experiment and their results. A possible scenario is described in the text box below. 


\section{Molly asks: How long is a day on Saturn?}

A seventh grade class is studying the Solar System. The diversity of the physical characteristics of the planets is a topic of discussion. The class is broken into nine groups, each assigned a planet. The Saturn group convenes and comes up with a list of questions to address. Molly is assigned the task of finding out the length of a Saturnian day. From within the VTIE portal, Molly searches the VTIE Journal for previous studies of Saturn and finds one paper on Saturn's rings. In the introduction, she comes across the work "albedo" which is new to her. She clicks on the word, and "a measure of the how much light is reflected from an object" appears in small pop-up window (had Molly been an undergraduate, "fractional reflectivity" would have appeared). Molly realizes she cannot simply look up the length of a day on Saturn in the VTIE Journal, so she will have to make a measurement. From her workspace within the VTIE portal, she invokes the Proposal Generation Tool to construct the following statement of the problem: I want to determine the rotation period of Saturn (See Figure 3). The Proposal Generation Tool consults the mapping between physical properties and observable quantities, and suggests to Molly two ways to determine rotation period: tracking and identifiable feature as it crosses the surface of the planet, and measuring the total light reflected from the planet as a function of time. Both of these methods require taking multiple images of the planet over several viewing sessions, and this is what Molly proposes to do. Her proposal is accepted. Over the course of a week, the class is awarded five two-hour chunks of observing time. Molly's group realizes that several questions asked in their group can be addressed by analyzing the same images, so they take control of the telescope three times during each two-hour slot and retrieve a total of 15 images during the week. Using the WebVTT analysis tool, Molly attempts to find the rotation period using both methods: first, she tracks a large oval surface feature as it crosses the surface of the planet, and secondly, she measures the total light reflected from the planet as a function of time. She finds the feature tracking easier to interpret, and concludes that the length of a day on Saturn is about half an Earth day. Molly invokes the Paper Writing Tool, and follows guidance that tells her what kind of information should go into the Introduction, Observations, Analysis and Conclusions section. She also uploads two of the Saturnian images, on which she has marked the position of the large oval. She submits the paper, and the teacher accepts it and it is entered into the VTIE Journal. Molly prints a PDF version of the paper to take home to her brother Hughie to show him that she has taken real pictures of the second largest planet in the Solar system, where a day is only half an Earth day long.

\section{SISTER Components}

SISTER comprises four basic components: a Glossary Tool (Item 1 in Figure 1) which adds instructional value to existing resources by adding automatically induced hyperlinks to an underlying glossary, the Proposal Generation Interface (Item 2 in Figure 1) which guides learners through the development of their astronomy observation request, Analysis Tools (Item 5 in Figure 1) for manipulating the data they obtain with the telescopes and elsewhere (Items 3 and 4 in Figure 1), and the Paper Writing Tool (Item 6 in Figure 1) which helps students organize their results and report their findings in a logical manner (Item 7 in Figure 1). The environment requires a standard Internet DHTML-enabled browser and an Adobe Acrobat Viewer, both of which are freely available for all major operating systems. The latter is required for viewing and printing 'professional-looking' Portable Document Format (PDF) versions of the student research papers; however, if the software is unavailable due to problems with installation, for example, then an HTML version may be produced and printed using the Internet Browser.

All resources (proposals, downloaded images, etc.) are stored centrally, and have a unique VTIE Uniform Resource Locator (URL) for ease of retrieval. The information flow in Figure 1 is essentially URL-based. The components are independent modules. The VTIE portal provides the users with a URL-naming, or aliasing, facility to identify specific URLs produced at various stages of the process. This allows the user to specify data sources by name or mnemonic, rather than a URL, which later may be subject to change due to upgrading or server/data re-location, etc. The VTIE portal will perform all name lookup and resolution and is independent of the SISTER process.

The four basic components of SISTER are described in more detail in this section.

\subsection{Glossary Tool}

Our goal is to provide access and add instructional value to the set of existing resources on the Web to stimulate student interest in a particular subject area. When accessed through the VTIE Portal, key terms in an exposition on a particular subject are hyperlinked to definitions in an underlying glossary by the VTIE Glossary Tool. The underlying glossaries are three tiered: middle school, high school, and advanced. The definitions returned are selected based on the student's user profile. 
For prototyping the software, we are using as content expositions the lesson plans developed in support of the original Telescopes in Education project. Clicking on the link to a particular exposition calls a CGI program (written in Perl) which scans the text of the exposition for terms that appear in the index of the glossary. When a match is found, a link is added from the term in the text to the glossary entry. The new page, comprising the original text and automatically induced hyperlinks, is delivered to the student.

The link induction process itself relies on a number of ad hoc parsing heuristics to identify the occurrence of a keyword or phrase. For example, "binary star" should be considered one term, rather than providing separate definitions for "binary" and "star." The link induction code will be documented and released in the Fall of 2002.

A number of companies provide software to automatically generate links to information resources in specified repositories, such as Microsoft's Smart Tags development kit which enables HTML documents to be scanned, for example, for company names, adding links to the company's home pages (http://msdn.microsoft.com/msdnnews/2001/may/SmartTag/Sm artTag.asp). Syntalex provides a more flexible product which allowed legal publishers Context Limited to dynamically link case law citations within legal documents to the most recent resources relevant to those citations [13]. Both the SmartTag and Syntalex systems can be used to link to glossaries, but they do not have inbuilt mechanisms to associate different target resource repositories to different classes of users. Although incorporating such customization should not be difficult, it is not possible without engaging the consulting services of the companies in question, since the software is closed source.

The only product we are aware of that dynamically adds links to different resources for different classes of users is the Context Sensitive Reference Linking system provided by SFX (http://www.sfxit.com). This system assumes that a user has subscribed to one or more digital libraries of information resources (e.g. legal publishing services, or citation services), and dynamically adds links to resources from the subscribed-to libraries. To identify subscribers, SFX relies on cookie session ids, which are similar to the non-cookie session ids that we use to identify the sophistication level of a user.

The OpenDictionary project at Stanford seeks to provide an infrastructure for automatic glossary linking [14], but does not provide the linking software itself. Rather, it is an effort to compile a contextually hierarchical dictionary, where each definition has its own home page, serving as a portal into the concepts that the definition embodies.

\subsection{Proposal Generation Interface}

After the students have explored ideas about astronomy using Web resources that have been "glossarized" as they pass through the linker in the VTIE portal, the problem-based learning approach dictates that they should formulate a question about an object of interest to them. While the set of possible questions is infinite, the choices available to the students are constrained by the types of measurements that can be made with the instrumentation available on the telescopes available for use by the students. Students are able to measure three quantities: apparent brightness (through a particular set of filters), morphology (angular size, shape), and, possibly, position on the sky relative to other known sources within the same CCD field. Thus, any questions asked by students must be related to these measurable quantities. To provide an interface that guides students to ask addressable questions, we have assembled an ontology of observation types for VTIE. This ontology includes a mapping of potential questions to corresponding observations.

Table 1: Mapping between Physical Properties and Observable Quantities

\begin{tabular}{|l|l|}
\hline Physical Property & Observable as Quantity \\
\hline Rotation period & Brightness a function of time \\
\hline Spectral Type & $\begin{array}{l}\text { Relative brightness through } \\
\text { certain filters }\end{array}$ \\
\hline Mass (of Jupiter) & $\begin{array}{l}\text { Position of moons as a } \\
\text { function of time }\end{array}$ \\
\hline $\begin{array}{l}\text { Distance to nearby galaxy } \\
\text { (e.g Andromeda) }\end{array}$ & $\begin{array}{l}\text { Brightness of Cephied } \\
\text { variables) over time }\end{array}$ \\
\hline
\end{tabular}

The first piece of the Proposal Generation Interface encountered by students is the Question Builder, which guides the students through the construction of their question (Figure 2). The students are able to choose from a set of physical properties they would like to study. The system references the ontology and dynamically provides the subsequent list of choices of celestial objects from which the students may choose. Once the students have formulated their question, the system once again references the ontology to map the physical property of interest into an observable quantity. Some examples of such mappings are given in Table 1. With time, the students should come to understand the mappings themselves. Using the suggestion from the Proposal Generation Interface, the student must come up with the proposal, which specifies celestial coordinates (or, in the case of non-sidereal objects, object name), filters to be used, instrument type (defaults to CCD), and, optionally, time and date. (The time and date are optional because 
most observations can be satisfied on multiple dates, though some events, such as eclipses, are clearly time dependent.)

The students submit their proposals, and the teacher reviews them and schedules the observations from within the VTIE portal. The telescopes are operated by software that is not part of VTIE, and, thus, that part of the process will not be discussed further in this paper.

\section{Question Builder Dynamic interface offering choices}

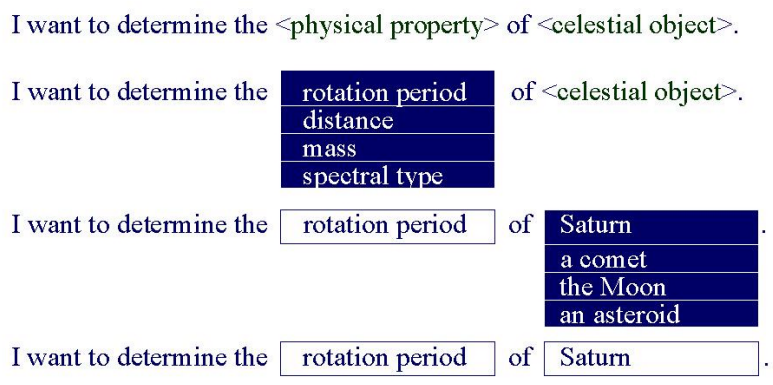

Figure 2: Proposal Generation Interface

\subsection{Analysis Tools}

The telescopes currently available in the TIE network provide the students with optical images of the sources they choose. VTIE, however, encourages the comparison of the optical observations with observations of the same celestial objects at various wavelengths (e.g. radio, infrared, X-ray), using an agent developed as part of the Telescope Resource Management component of VTIE [7]. This agent retrieves images of the source from SkyView (http://skyview.gsfc.nasa.gov). Using some custom and some generic image manipulation tools, the students are able to compare and contrast the morphology of the sources in the various wavelength bands. Ultimately, the goal is to help the students understand that different wavelength regimes provide information on different physical processes.

At the time of writing, VTIE is using the Visual Target Tuner, a simple image analysis tool developed in the Advanced Automation Branch NASA Goddard Space Flight Center. Soon a browser-enabled version of this tool, WebVTT, will be available. Using this tool, students import their optical image and one other image (e.g. from SkyView) and do simple plotting and labeling on the images. The results of the image analysis is stored and accessed via URLs by the tool for display and will be imported later by the Paper Writing Tool. In the second release of VTIE, a more comprehensive (but still webbased) image analysis package will be included.

\subsection{Paper Writing Tool}

The students will report their results using the Paper Writing Tool (Figure 3). Upon successful review by the teachers, these papers will become part of the online VTIE Journal. The students are presented with a dynamically-generated HTML form which contains all of the elements of a typical paper, for example Title, Abstract, Keywords, Introduction, Observations, Analysis, Conclusions, Figures, and References. The general layout for the paper is provided to focus the students on writing rather than on formatting the document. Save, Revert and Submit options will be provided so that the report could be started during one class period and completed during another or at home. By choosing the Submit option, a student 1) sends the paper to a reviewer, as do professionals (in TIE, usually the teacher or a combination of the teacher and the author's peers) and 2) stores the components of the paper (each separately tagged as XML). All VTIE Journal papers will be viewable in PDF format. These are final documents which will become a public resource to the VTIE and non-VTIE community (for example, to be used in the very first step of the process by subsequent students).

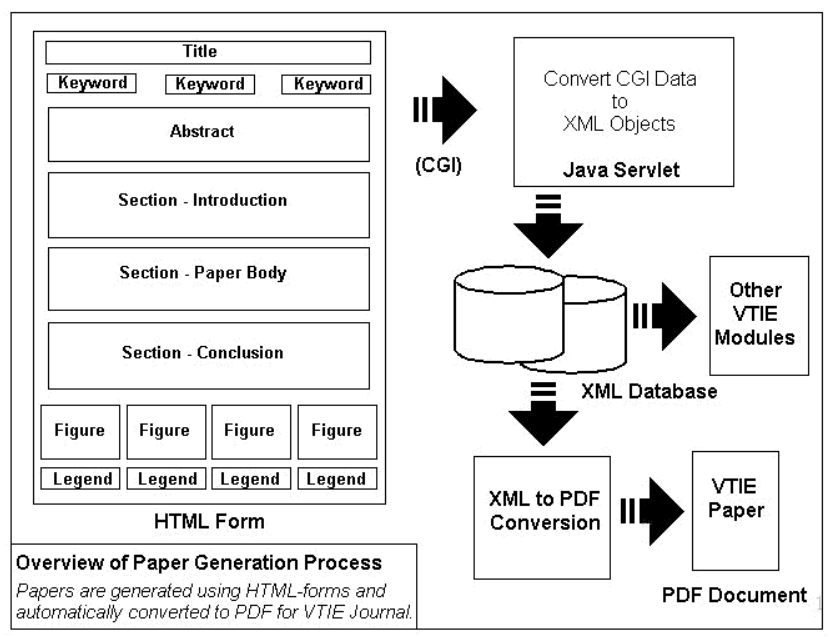

Figure 3: Paper Generation Tool

\subsection{Implementation}

Both the Proposal Generation Interface and Paper Writing Tool currently use non-Cascading Style Sheet, browser independent, DHTML to implement the user interface. JavaScript is used to perform validation prior to form submission where necessary and to script the various form elements used to construct the interface. For 
example, in the Proposal Generation Interface, user choice (for celestial object) is currently implemented using <SELECT> tags within a form. The content (options) of the first <SELECT $>$ tag (physical property) is currently fixed, but scripted to dynamically update the options in the second $<$ SELECT $>$ tag depending on the option that is selected. This dynamic update works very well, although in Internet Explorer, form elements 'reflow' during update, which tends to temporarily disturb the layout of the user interface. There are also problems with <OPTION $>$ tag scripting using Netscape Navigator due to the fact that the form elements do not re-flow during update. In particular, the width of the <SELECT $>$ tag remains constant after it is first rendered. Thus, nonvisible selections may only become partially visible during the interaction process. Therefore, the width of all elements must be known in advance and the elements adjusted accordingly at the outset.

We are currently implementing a browser independent CSS-based menu interface that should overcome these issues, although it introduces the drawback that the new interface will only operate in a CSS-enabled browser. Although the current document is static, we plan to replace it with a Java Server Page (JSP) allowing both dynamic page generation suitable for the user's browser type and user session tracking. All of the tools in the SISTER environment use Perl to implement server processing, but during the Summer of 2002, almost software will be written in Java. At this stage in the VTIE development process, there are many technologies used, and we have made a strategic decision to implement a homogenous system using Java. Our main motivation is the continuing maintenance of the system, and the complication introduced when maintaining heterogeneous systems.

The Proposal Generation Interface uses a form to enable students to specify their proposal, and all form elements are submitted to a server-based (Perl) CGI processing back-end. The content of the proposal, including the initial scientific question, the observation specifications and the contents of a $<$ TEXTAREA $>$ form element where students explain their experiment, is tagged using XML and stored in a database for later use. In particular, the celestial object and observing coordinates will become available for the scheduler (part of the VTIE system) and the proposal content will become available for the Paper Writing Tool. Upon submission, the teacher will examine the proposal, and if the proposal is considered appropriate, it will be approved and the student may move onto the next step. If there are problems (grammar, poor thesis, etc.) then the proposal will be returned to the student with appropriate comments. This process continues until the proposal is approved. A $\log$ of all changes and transaction is maintained in the database by VTIE. Note that the document never 'leaves' the VTIE environment, and neither the teacher nor the student requires additional text development tools - everything takes place within the browser.

For storing XML data, SISTER uses the XINDICE native XML database. The use of native XML storage has several advantages. First, the database schema is easily conceived as a collection of micro-schemas representing the various SISTER components. Second, there is a direct correspondence between the schema and the forms used in the Proposal Generation and Paper Writing tools, so the schema drives the forms. Third, XML elements can be easily selected to generate custom documents. Finally, VTIE data, consisting of parent elements which determine the nature of child elements, is hierarchical (rather than tabular) in nature, and thus is more appropriate in an XML database than in a relational system.

The Paper Writing Tool also uses session tracking and storage of content to the XML database. This allows the content to be viewed by the teacher in HTML format and allows the mentor to introduce comments (also stored as XML) on the student paper, following the same approval process as for the original observing proposals. Once the paper is approved, all comments are removed, and the resulting content is used to produce either a HTML (for online viewing online) or a PDF (for printing) document. The paper is automatically incorporated into the online VTIE journal, and the various indices updated. In order to create the PDF document, the server program (written in Perl) uses a Latex template, which is passed to a 'PDFLatex' processor to produce a PDF document. This processor analyses the size of all images and performs appropriate scaling and placement of images in the paper. Currently, it also performs image type conversion from GIF format to PNG format, which is the standard format for all images rendered by the VTIE environment. (VTIE stores the FITS format images from the telescope for use with astronomy processing software.)

\section{Status}

The Virtual Telescopes in Education project launched alpha testing at the Science Camp at the National University of Ireland Maynooth in July 2002. The system was deployed to six groups of 20 - 25 high school students. The sessions were two hours each. Three groups remotely operated the 24-inch Mt. Wilson telescope in California to obtain an optical image. Three groups obtained their optical images from an online archive. Each group compared the optical image with an 
image of their source in a different wavelength regime. Each group prepared a short summary of their results. This process was video taped and observed in real-time by six observers.. The video tapes and the reports are being analyzed to determine 1) the usability of the software and 2) the level of understanding of the science process by the students.

Results from this testing will be incorporated into the prototype to be launched in the fall of 2002 and will be reported at HICSS 36. Initial results from the fall deployment will also be available for presentation at the conference.

\section{Acknowledgments}

This material is based upon work supported by the National Science Foundation under Grant No. 0121531. Our thanks to the National University of Ireland for supporting VTIE participation in Science Camp 2002.

\section{References}

[1] S. Hoban, M. desJardins, N. Farrell, P. Rathod, J. Sachs, S. Sansare, Y. Yesha, J. Keating, B. Busschots, J. Means, G.

Clark, L. Mayo and W Smith (2002), "Virtual Telescopes in

Education," Journal of Digital Information, 2 (4)

(http://jodi.ecs.soton.ac.uk/Articles/v02/i04/Hoban/).

[2] J. E.F. Baruch (1993), "Robots in Astronomy," Vistas in Astronomy, 35, 399-438.

[3] J. E.F. Baruch (2000), "The Future of Robotic Telescopes for Education," Publications of the Astronomical Society of Australia, 17 (2), 119

(http://www.atnf.csiro.au/pasa/17 2/baruch/).
[4] D. McKinnon and H. Geissinger (2002), "Interactive Astronomy in Elementary Schools," Educational Technology \& Society, 5 (1)

(http://ifets.gmd.de/periodical/vol 1 2002/mckinnon.HTML).

[5] B. Grabowski, T.A. Koszalka, and Y. Kim (2002), "Designing Problem-Based Learning with Web Resources to Inspire Middle School Kids: KaMS (Kids as Airborne Mission Scientists)," Educational Technology Research and Development (submitted).

[6] M. desJardins, P. Rathod, and S. Sansare, (2002), "Interactive, Incremental Scheduling for Virtual Telescopes in Education," Third International NASA Workshop on Planning and Scheduling for Space, Houston, Texas, October 2002 (in press).

[7] Busschots, B., J. Keating, and S. Hoban (2002), "Independent Agents in the VTIE Telescope Resource Management System," Information Sciences (submitted).

[8] F. T. Hofstetter (1995). Multimedia Literacy, New York: McGraw-Hill.

[9] M. Neo and K. T. K. Neo (2001). "Innovative teaching: Using multimedia in a problem-based learning environment," Educational Technology \& Society 4 (4) (http://ifets.ieee.org/periodical/vol 4 2001/neo.HTML).

[11] B. Bloom (1956) Taxonomy o Educational Objectives, Handbook I: Cognitive Domain. New York.

[12] L. Allen (2001) Competencies That Count: Strategies for assessing high-performance skills.

[13] J. Needle (2002) "The Automatic Linking of Legal Citations," http://elj.warwick.ac.uk/jilt/00-3/needle.HTML

[14] J.O. Smith, III (2002) “The Open Dictionary - Automatic Linking", http://ccrmawww.stanford.edu/ jos/od/Automatic Linking.HTML 